\title{
Feasibility of the Reconstruction with Fascia Lata Patch on the Abdominal Wall Defect After Resection of the Abdominal Desmoid Tumor
}

This article was published in the following Dove Press journal:

Clinical and Experimental Gastroenterology

\section{Ayana Goto \\ Nobuhisa Matsuhashi \\ Takao Takahashi \\ Toshiyuki Tanahashi \\ Satoshi Matsui \\ Hisashi Imai \\ Yoshihiro Tanaka \\ Kazuya Yamaguchi \\ Kazuhiro Yoshida}

Department of Surgical Oncology, Gifu University School of Medicine, Gifu City 50I-II94, Japan
Correspondence: Nobuhisa Matsuhashi Department of Surgical Oncology, Gifu University School of Medicine, I-I Yanagido, Gifu City 50I-I 194, Japan

Tel +8I-58-230-6233

Fax +8I-58-230-1074

Email nobuhisa517@hotmail.com
Introduction: The abdominal desmoid tumor shows invasive development and high local recurrence rate. The primary treatment method is complete removal of the tumor because of the high recurrence rate; however, the problem for the surgeon is the reconstruction of the abdominal wall after resection of the abdominal desmoid tumor.

Case Presentation: A 63-year-old man underwent open drainage and ileostomy for the perforation of ileocecal tumor. After 3 months, he underwent right hemicolectomy and ileostomy closure. Pathological examination revealed no malignancy, and the ileocecal tumor showed the presence of abscess. He noticed a palpable mass in the left abdomen. Enhanced abdominal computed tomography (CT) revealed a large abdominal incisional hernia and an enhanced mass of $40 \mathrm{~mm}$ in the left rectus muscle. Needle biopsy was performed and the diagnosis was desmoid tumor. He underwent resection of the desmoid tumor and repair of hernia. We performed wide local resection, with a 2-cm surgical margin. The hernia was repaired by simple closure, and the defect in the left abdomen was repaired with reconstruction using the fascia lata patch through plastic surgery.

Conclusion: We encountered a case of abdominal wall desmoid tumor combined with a large abdominal incisional hernia. We selected the use of autologous fascia based on the risk of recurrence. The patient has not shown recurrence of incisional hernia or desmoid tumor 22 months after surgery. The use of fascia lata patch can be considered as a satisfactory alternative for such reconstruction cases.

Keywords: abdominal desmoid tumor, incisional hernia, abdominal wall reconstruction, autologous fascia

\section{Introduction}

Desmoid tumor is a benign tumor that arises from the myofascial and musculosaurus tissues. This tumor is rare, with an estimated incidence rate of "2.4"-"4.3" per 1 million population. ${ }^{1}$ They do not exhibit distant metastasis; however, the tumor tends to strongly infiltrate neighboring tissues, resulting in local recurrence. ${ }^{2}$ The rate of local recurrence is $25 \%-50 \%{ }^{3,4}$ The primary treatment method is complete removal of the tumor to control local recurrence, although surgeons face problems in the reconstruction of the abdominal wall defect after resection.

\section{Case Presentation}

A 63-year-old man attended our hospital with heavy abdominal pain in January 2016. The CT revealed a mass with elevation of tissue fascia and free air 


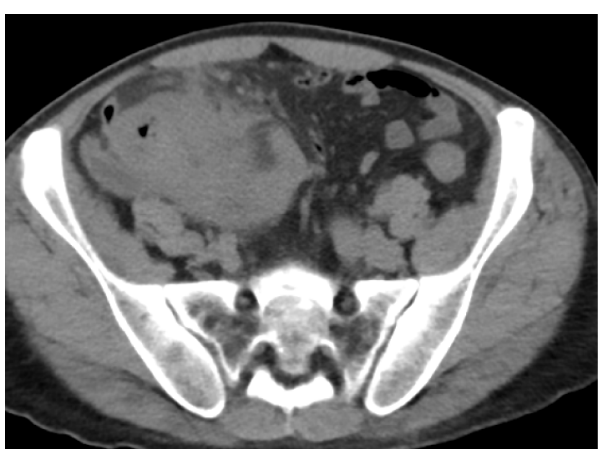

A

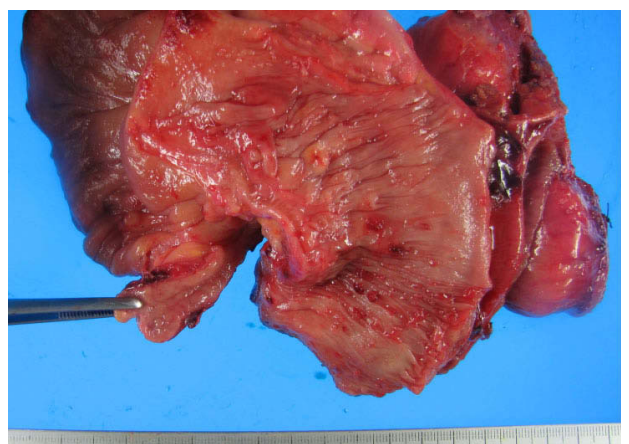

B

Figure I There was a mass near the cecum. The elevation of tissue fascia and free air were detected (A). There were several small polyps at ileocecal section (B).

near the cecum (Figure 1A). Accordingly, the patient was diagnosed with perforation of the cecal diverticulum, and open drainage and construction of ileostomy was performed. After 3 months, the patient received right hemicolectomy and closure of the ileostomy. We did not detect the mass from the resected specimen (Figure 1B), and pathological examination revealed no malignancy.

The patient experienced a palpable mass in the left abdomen from May 2017 and attended our department in December 2017. The enhanced CT revealed a $38-\mathrm{mm}$ mass, resembling a dyeing ring in the left rectus abdominis muscle. The mass was located near the scar of ileostomy closure (Figure 2). The size of hernia orifice from the epigastrium to the pubic bones was approximately $20 \mathrm{~cm}$.

After 4 months, the MRI revealed a $40-\mathrm{mm}$ mass in the left rectus abdominis muscle. T2 weighted image

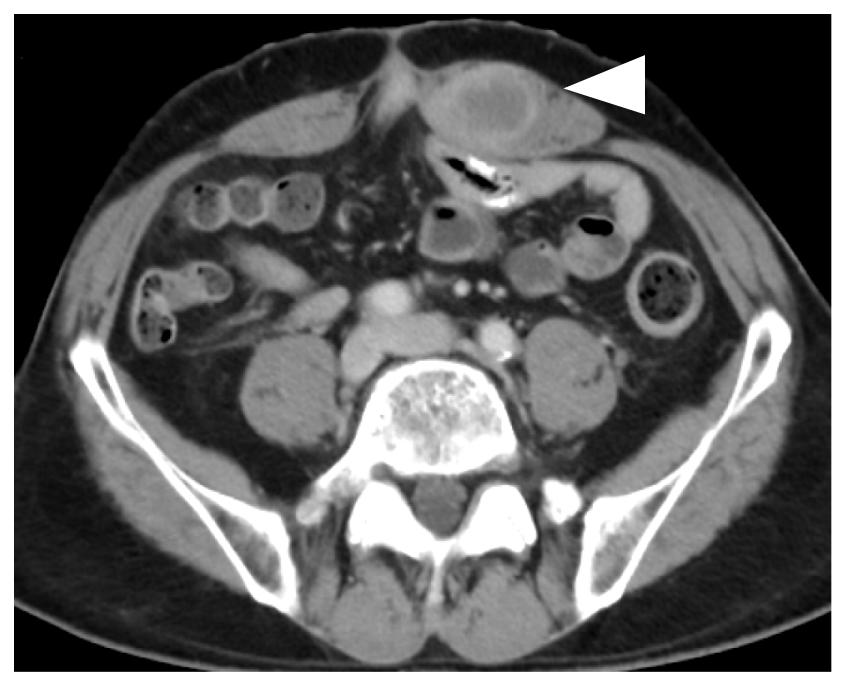

Figure 2 The enhanced CT in December 2017 showed the mass of $38 \mathrm{~mm}$ with dyeing ring form in the left rectus abdominis muscle. The mass was located near the ileostomy closure scar. The triangle points to the tumor. showed a low intensity area similar to muscle (Figure 3A), and the tumor was uniformly enhanced (Figure 3B).

We performed endoscopic ultrasound (EUS) and fine needle aspiration of the mass and reached the pathological diagnosis of desmoid tumor. Because the patient had pain at the mass and at the point of abdominal incisional hernia, we planned resection of the abdominal desmoid tumor and repair of incisional hernia.

We performed a midline incision similar to the previous scar and cut a spindle shape around the mass at $2-\mathrm{cm}$ margin from the mass (Figure 4A). There was no exposure of the tumor at the abdominal side. We used the Harmonic Scalpel $^{\mathrm{TM}}$ (Ethicon Endo-Surgery, Cincinnati) to cut the muscle to prevent bleeding (Figure 4B). Next, we removed the rectus abdominis muscle from the left lateral end. The abdominal wall defect measured $10 \mathrm{~cm} \times 10 \mathrm{~cm}$ (Figure 4C). The hernia was repaired with a simple closure using anterior layer of rectus sheath and the defect was closed by suturing the right anterior layer of rectus sheath to the left aponeurosis of external oblique muscle (Figure 4D). Considering the risk of recurrence of abdominal wall hernia at the defect site, we decided to repair the defect with autologous fascia. We used a fascia lata patch measuring $15 \mathrm{~cm} \times 5 \mathrm{~cm}$ (Figure 4E) to repair the defect in the left abdomen (Figures $4 \mathrm{~F}$ and $5 \mathrm{~A}-\mathrm{C}$ ).

The size of the mass was $45 \mathrm{~mm} \times 45 \mathrm{~mm}$, and it was a solid tumor. The macroscopic view of the resected tumor revealed its whitish color (Figure 6A). Pathological analysis revealed the growth of fibroblasts, defect of the nuclear heteromorphism, and presence of abundant collagen fibers between fibroblasts (Figure 6B). Additionally, a nuclear-positive image of $\beta$-catenin was observed on immunostaining (Figure 6C). The 


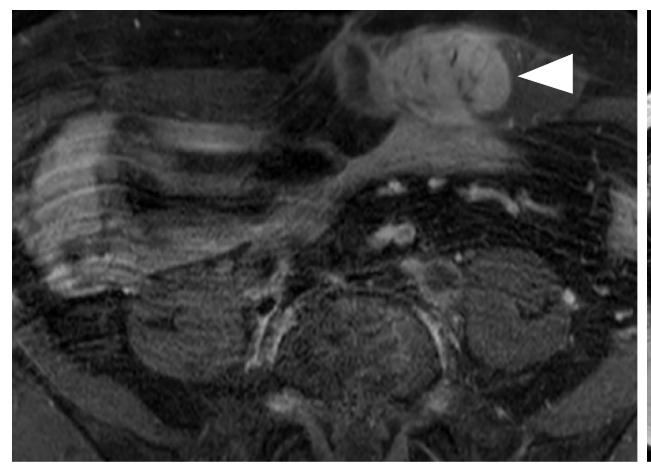

A

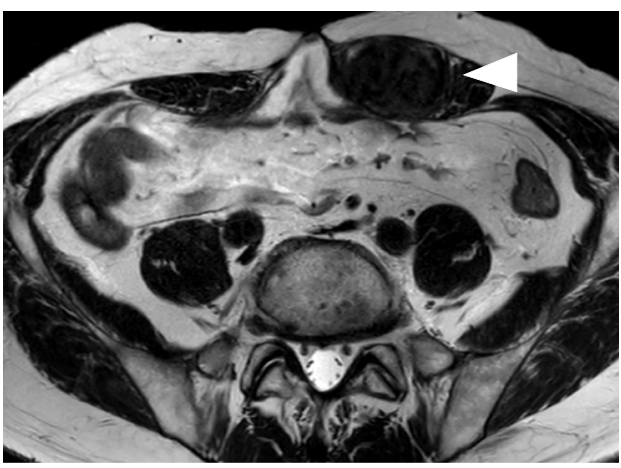

B

Figure 3 The MRI in April 2018 showed the mass of $40 \mathrm{~mm}$ in the left rectus abdominis muscle. The mass showed low intensity area like muscle in T2 weighted images (A). It was uniformly enhanced (B). The triangle points to the tumor.

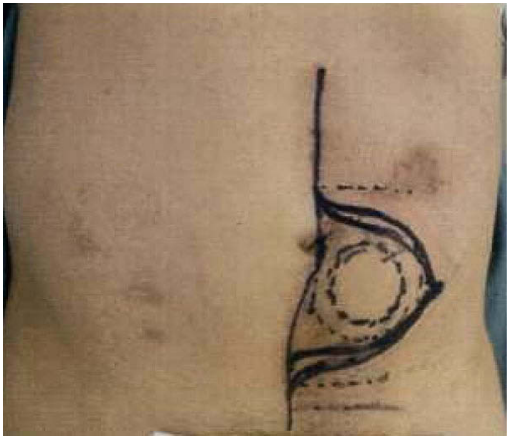

A

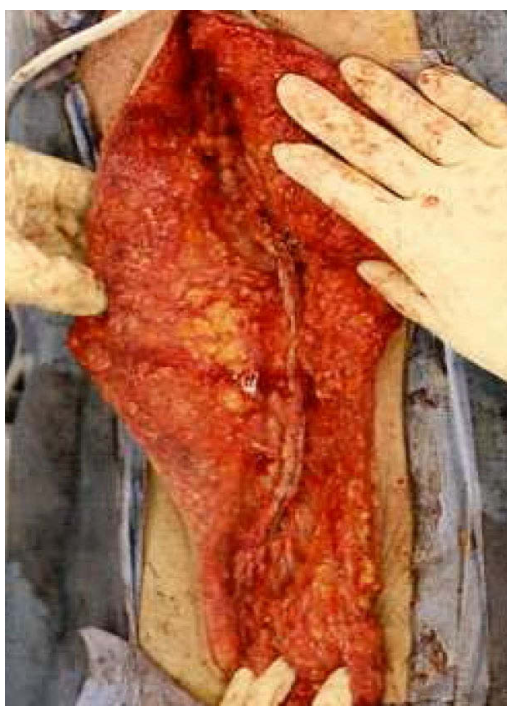

D

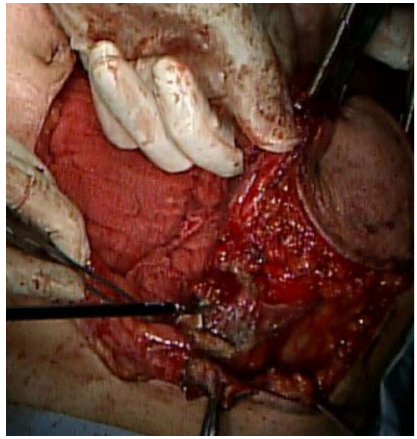

B

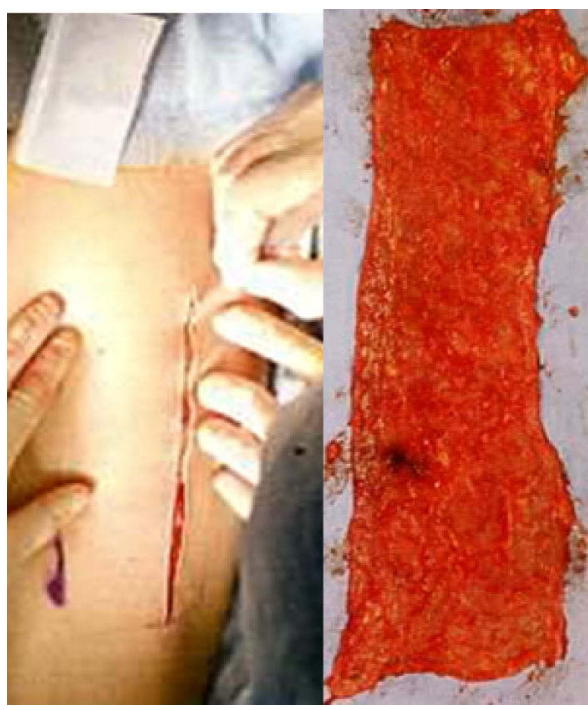

E

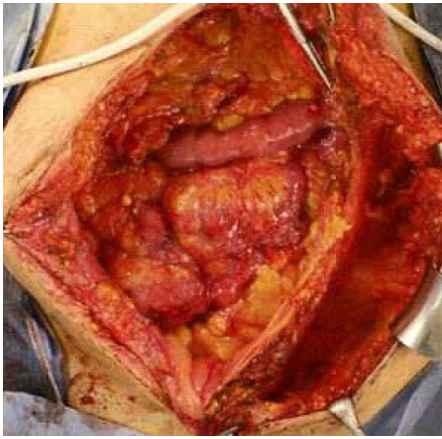

C

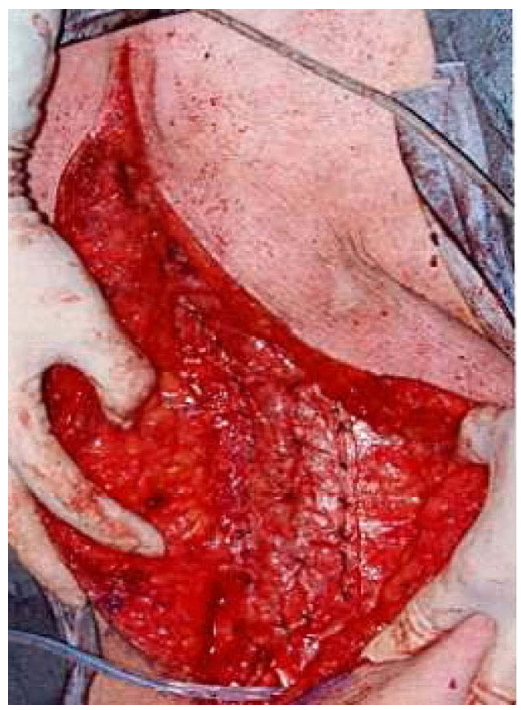

$\mathbf{F}$

Figure 4 We performed a midline incision similar to the previous scar and we cut a spindle shape around the mass at $2 \mathrm{~cm}$ margin from the mass (A). There was no exposure of the tumor to abdominal side. We used ultrasonically activated scalpel when we cut the muscle (B). Next, we removed the rectus abdominis muscle from the left lateral end. The abdominal wall defect was $10 \mathrm{~cm} \times 10 \mathrm{~cm}(\mathbf{C})$. The hernia was repaired with a simple closure using anterior layer of rectus sheath and the defect was closed by suturing the right anterior layer of rectus sheath to the left aponeurosis of external oblique muscle (D). We used a fascia lata patch measuring $15 \mathrm{~cm} \times 5 \mathrm{~cm}(\mathbf{E})$ to repair the defect in the left abdomen (F). 


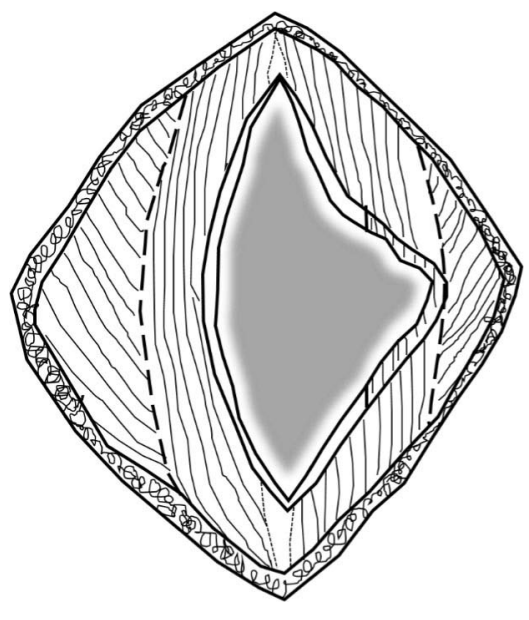

A

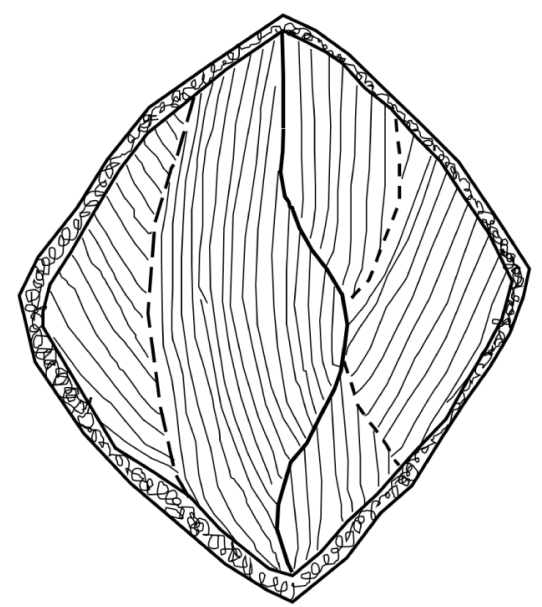

B

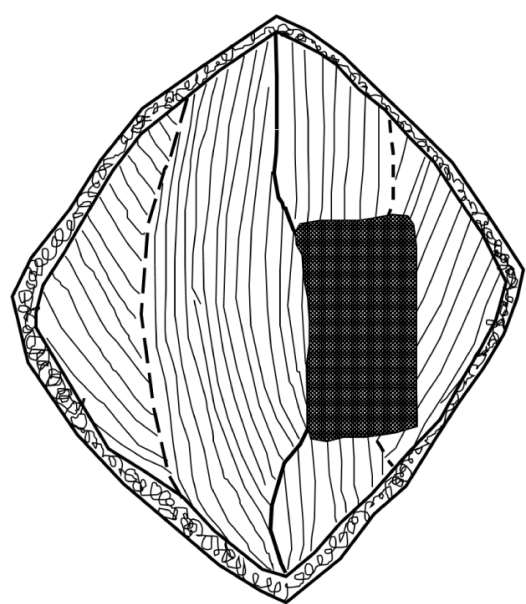

C

Figure $\mathbf{5}$ The final form after resection of tumor $(\mathbf{A})$. The gray zone is the part that was removed. The defect was repaired with a simple closure and the defect was closed by suturing the right anterior layer of rectus sheath to the left aponeurosis of external oblique muscle (B). We used fascia lata patch as on lay mesh on defect (C).

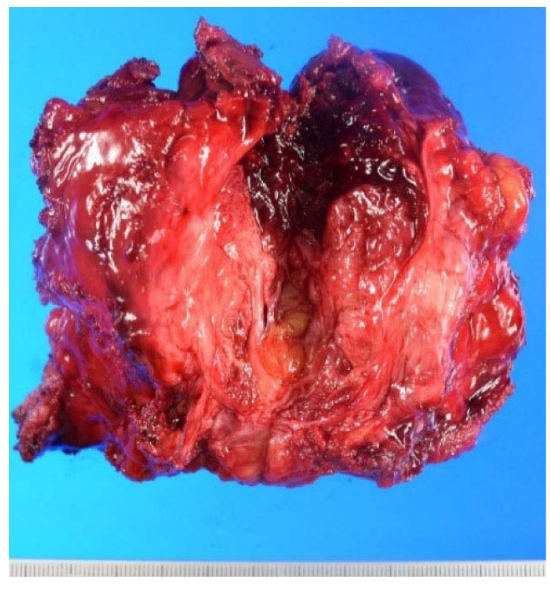

A

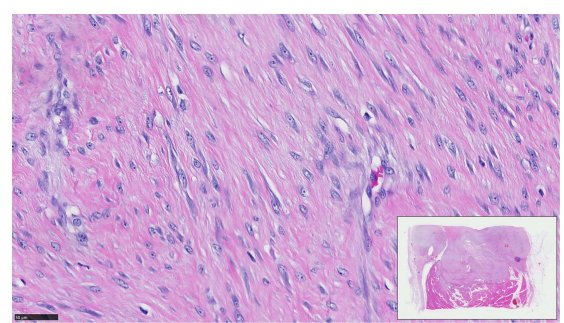

B

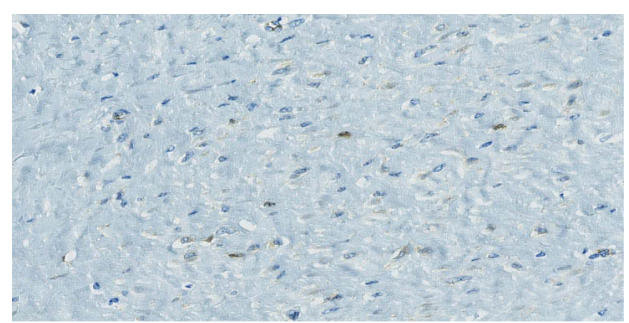

C

Figure 6 The tissue image is a magnified view of the red square part in the lower right image. The black line at the bottom left shows $50 \mu \mathrm{m}$. The size of the mass was $45 \mathrm{~mm} \times 45 \mathrm{~mm}$, the cut surface was white $(\mathbf{A})$. The growth of fibroblasts was seen, the nuclear heteromorphic was poor. There was abundant collagen fibers between fibroblasts (B). A nuclear positive image of $\beta$-Catenin was observed by immunostaining (C).

pathological diagnosis was desmoid-type fibromatosis. No recurrence of incisional hernia and desmoid tumor was detected 22 months after surgery.

\section{Discussion}

Desmoid tumor is a benign tumor that arises from the myofascial and musculosaurus tissues, with an estimated 
incidence of $2.4-4.3$ per 1 million population. ${ }^{1}$ It has the following clinicopathological features: i) it is rich in differentiated fibroblasts, ii) it has plenty of collagen fibers between fibroblasts, iii) it is not malignant, iv) it tends to strongly infiltrate, and v) it does not show distant metastasis, although local recurrence can occur. ${ }^{2}$ The rate of local recurrence has been reported to be $25-50 \%{ }^{3,4}$ The association with FAP, pregnancy, parturition, trauma, and surgical history are considered as risk factors. It is diagnosed by using imaging technologies such as ultrasonography, CT, or MRI. The definitive diagnosis is based on pathological findings such as growth of fibroblasts with collagen fibers between cells.

The primary treatment method is complete removal to prevent local recurrence. Regarding the setting of the resection line, no specific consensus has yet been shown. Rock et al reported the recurrence rate of $68 \%$ in overall resected cases, $48 \%$ in extensive resection cases, and $90 \%$ in marginal resection cases.5 Gronchi et al reported that the recurrence rate is unaffected by whether the margin is positive or negative. ${ }^{6}$ Other studies ${ }^{7}$ have reported that tumor size, age, and occurrence location are predictors.

The methods employed for the treatment of desmoid tumor have changed in the past 5-10 years because surgery itself can be a risk of recurrence and cause complications. Recently, conservative approaches such as pharmacological or simple observation have been selected as treatment. ${ }^{7}$

After the resection of abdominal desmoid tumor, the problem is reconstruction of the abdominal wall defect. Regarding the method of reconstruction of the abdominal wall, besides performing primary closure or using artificial materials such as mesh, reconstruction via transplantation of flap or autologous fascia is selected. The materials used for fascia include anterior layer of rectus sheath, aponeurosis of external oblique muscle, and fascia lata patch. The fascia lata patch is generally preferred because we can easily get resilient and large fascia. ${ }^{8}$ Yamamoto studied cases using fascia lata patch as an onlay mesh to reinforce fascial closure when they overlapped with the defect in the anterior rectus fascia. They found no evidence of recurrence in the median follow-up time of 16.1 months. ${ }^{9}$ Stecco et al described that the fascia lata could be used alone as a mesh and that it is more easily integrated into the new site than an artificial mesh. ${ }^{10}$ Hamilton microscopically observed a piece of a patient's fascia lata patch that was removed 2 years after insertion. They confirmed the presence of dense wavy layers of collagen and numerous small nutrient vessels. ${ }^{11}$ Thus, the fascia lata patch can repair the defect if the reconstruction is only supportive tissue. It was considered to be useful as a mesh when using autologous tissues, particularly when the use of artifacts was not desired. In addition, fascia lata patch technique is easily performed and comfortable for gastrointestinal surgeons.

In this case, we wanted to achieve complete resection, repair of the large incisional hernia, and reconstruction of the abdominal defect after resection of desmoid tumor. Therefore, we selected a margin of $2 \mathrm{~cm}$ and decided to use the fascia lata patch based on the risk of future recurrence. Although the follow-up period is short, we did not experience recurrence of incisional hernia.

\section{Conclusion}

We have reported a case of abdominal wall desmoid tumor occurring in combination with a large abdominal incisional hernia. In this case, the tumor could have been caused owing to the history of abdominal surgery. Therefore, considering the risk of recurrence in the future, we used fascia lata patch for reconstruction of the abdominal defect. Although we considered the use of a fascia lata patch to be a useful technique for patients with large incisional hernia, a multicenter trial is deemed necessary to evaluate the risks and benefits of using fascia lata patch.

\section{Ethics Approval and Informed Consent}

We obtained permission from the ethics committee of Gifu University school of medicine for this case report.

\section{Consent for Publication}

Written informed consent was obtained from the patient for publication of this case report and accompanying images.

\section{Acknowledgments}

We thank plastic surgeons Yuji Yasue, Keishi Koyama, Hisakazu Kato at the Department of Plastic and Reconstructive Surgery, Gifu University School of Medicine for participating in this operation.

\section{Author Contributions}

All authors contributed to data analysis, drafting or revising the article, gave final approval of the version to be published, and agree to be accountable for all aspects of the work. 


\section{Funding}

Funding information is not applicable.

\section{Disclosure}

T. Takahashi has received honoraria for lectures from Takeda Pharmaceutical Co., Ltd.All remaining authors declare that they have no conflict of interest. Dr Kazuhiro Yoshida reports grants, personal fees from Taiho Pharm, grants, personal fees from Asahi Kasei Pharma, grants, personal fees from Chugai Pharm., grants, personal fees from Covidien Japan, grants, personal fees from Daiichi Sankyo, grants, personal fees from Eisai, grants, personal fees from Eli Lilly Japan, grants, personal fees from Johnson \& Johnson, grants, personal fees from MerkSerono, grants, personal fees from MSD, grants, personal fees from Nippon Kayaku, grants, personal fees from Novartis, grants, personal fees from Ono Pharm., grants, personal fees from Otsuka Pharm., grants, personal fees from Sanofi, grants, personal fees from Tsumura, grants, personal fees from Yakult Honsha, grants from Abbott, grants from Abbvie, grants from Astellas, grants from Biogen Japan, grants from Celgene, grants from GlaxoSmithKline, grants from KCI, grants from Kyowa Kirin, grants from Meiji Seika Pharma, grants from Toray Medical, grants from AstraZeneka, personal fees from Denka, personal fees from EA Pharma, personal fees from Olympus, personal fees from Pfizer, personal fees from Bristol-Myers Squibb, personal fees from Sanwa Kagaku Kenkyusho, personal fees from SBI Pharma, grants, personal fees from Takeda Pharm., personal fees from TeijinPhamra, personal fees from TERUMO, grants from Koninklijke Philips, outside the submitted work. Dr Takao Takahashi has received honoraria for lectures from Takeda Pharmaceutical Co., Ltd. All remaining authors declare that they have no conflicts of interest.

\section{References}

1. Reitamo JJ, Häyry P, Nykyri E, Saxën E. The desmoids tumor. I. Incidence, sex-, age-, and anatomical distribution in the Finnish population. Am J Clin Pathol. 1982;77:665-673. doi:10.1093/ajcp/ 77.6.665

2. Stout AP, Latters R. Tumors of the Soft Tissues. Atlas of Tumor Pathology, 2nd Series, Fascicle 1. Washington DC: Armed Forces Institute of Pathology; 1967:17-22.

3. Easter DW, Halasz NA. Recent trends in the management of desmoid tumors: summary of 19 cases and review of the literature. Ann Surg. 1989;210:765-769. doi:10.1097/00000658-198912000-00012

4. Waddel WR, Gerner RE. Indomethacin and ascorbate inhibit desmoid tumors. J Surg Oncol. 1990;15:85-90. doi:10.1002/jso.2930150113

5. Rock MG, Pritchard DJ, Reiman HM, Soule EH, Brewster RC. Extra-abdominal desmoids tumor. $J$ Bone Joint Surg Am. 1984;66:1369-1374. doi:10.2106/00004623-198466090-00007

6. Gronchi A, Casali PG, Mariani L, et al. Quality of surgery and outcome in extra-abdominal aggressive fibromatosis: a series of patients surgically treated at a signal institution. J Clin Oncol. 2003;21:1390-1397. doi:10.1200/JCO.2003.05.150

7. Couto Netto SD, Teixeira F, Menegozzo CAM, et al. Sporadic abdominal wall desmoid type fibromatosis: treatment paradigm after thirty two years. BMC Surg. 2018;18:37. doi:10.1186/s12893-0180367-6

8. Ogino A, Onishi K. Reconstruction of abdominal wall hernia using autologous fascia graft. Surgery. 2018;80:222-225.

9. Miyamoto Y, Watanabe M, Ishimoto T, et al. Fascia lata onlay patch for repairing infected incisional hernias. Surg Today. 2015;45:121-124. doi:10.1007/s00595-014-0936-y

10. Stecco C, Tiengo C, Stecco A, et al. Fascia redefined: anatomical features and technical relevance in fascial flap surgery. Surg Radiol Anat. 2013;35:369-376. doi:10.1007/s00276-012-1058-0

11. Hamilton JE. Free mattressed fascia lata patches in the repair of large or difficult hernias: a 16-year experience. Ann Surg. 1963;157:925-931. doi:10.1097/00000658-196306000-00011
Clinical and Experimental Gastroenterology

\section{Publish your work in this journal}

Clinical and Experimental Gastroenterology is an international, peerreviewed, open access, online journal publishing original research, reports, editorials, reviews and commentaries on all aspects of gastroenterology in the clinic and laboratory. This journal is indexed on American Chemical Society's Chemical Abstracts Service (CAS).
The manuscript management system is completely online and includes a very quick and fair peer-review system, which is all easy to use. Visit http://www.dovepress.com/testimonials.php to read real quotes from published authors. 